\title{
Temperature dependence of the direct band gap of Si-containing carbon nitride crystalline films
}

\author{
D. Y. Lin, C. F. Li, and Y. S. Huang* \\ Department of Electronic Engineering, National Taiwan Institute of Technology, Taipei 106, Taiwan, Republic of China \\ Y. C. Jong and Y. F. Chen \\ Department of Physics, National Taiwan University, Taipei 106, Taiwan, Republic of China \\ L. C. Chen and C. K. Chen \\ Center for Condensed Matter Sciences, National Taiwan University, Taipei 106, Taiwan, Republic of China \\ K. H. Chen and D. M. Bhusari \\ Institute of Atomic and Molecular Sciences, Academia Sinica, Taipei 106, Taiwan, Republic of China
}

(Received 27 March 1997)

\begin{abstract}
We have measured the temperature dependence of the spectral features in the vicinity of the direct gap $E_{g}^{d}$ of Si-containing carbon nitride polycrystalline films in the temperature range between 20 and $500 \mathrm{~K}$ using piezoreflectance (PzR). From a detailed line-shape fit to the PzR spectra, the $E_{g}^{d}$ and the broadening parameter of direct band-to-band transitions at various temperatures are determined. The temperature dependence of $E_{g}^{d}$ are analyzed by the Varshni equation [Physica 34, 149 (1967)] and an empirical expression proposed by O'Donnel and Chen [Appl. Phys. Lett. 58, 2924 (1991)]. The parameters that describe the temperature dependence of the energy gap of the material are evaluated and discussed. The broadening parameter is found to be insensitive to the temperature variation. [S0163-1829(97)01135-X]
\end{abstract}

Carbon nitride films have attracted the attention of various investigators due to their interesting characteristics and wide range of promising application such as cutting tools, wear resistance, and a hard barrier against corrosion. ${ }^{1}$ Liu and Cohen ${ }^{2,3}$ predicted through an empirical model that covalent solids formed between carbon and nitrogen would be good candidates for extreme hardness (comparable to or greater than that of diamonds), and that these materials could be synthesized in the laboratory. However, it must be noted that although initial calculations have been performed only for $\beta-\mathrm{C}_{3} \mathrm{~N}_{4}$, it may not be the only stable structure for a covalent $\mathrm{C}-\mathrm{N}$ crystalline solid. In fact, more recent theoretical studies $^{4,5}$ have also investigated the stability and properties of $\mathrm{C}_{3} \mathrm{~N}_{4}$ compounds that have structures distinct from the $\beta-\mathrm{C}_{3} \mathrm{~N}_{4}$ structure. For instance, the results of Guo and Goddard III $^{6}$ calculations have indicated that $\alpha-\mathrm{C}_{3} \mathrm{~N}_{4}$ would be more stable than $\beta-\mathrm{C}_{3} \mathrm{~N}_{4}$. Actually, any $\mathrm{C}-\mathrm{N}$ covalent solid comprised of tetrahedrally bonded carbon atoms is likely to be very hard due to the small atomic sizes of carbon and nitrogen, low ionicity of the $\mathrm{C}-\mathrm{N}$ bond, and sufficiently high coordination number. Despite their technological importance, very few experimental studies on the fundamental physical properties of the $\mathrm{C}-\mathrm{N}$ bond materials have appeared in the literature, presumably due to lack of samples for quantitative measurements. Recent progress in growth techniques has enabled us to synthesize large Si-containing polycrystalline carbon nitride $(\mathrm{SiCN})$ films, ${ }^{7,8}$ enabling a reliable study of the physical properties of these interesting materials.

In this paper we report a study of the temperature dependence of the spectral features in the vicinity of the direct gap $E_{g}^{d}$ of the SiCN polycrystalline films in the temperature range between 20 and $500 \mathrm{~K}$ using piezoreflectance. PzR has been proven to be useful in the investigation and characterization of semiconductors. ${ }^{9,10}$ From a detailed line-shape fit we have been able to accurately determine the temperature dependence of the direct band gap and the broadening parameter of the direct band-to-band transitions of the material. We have analyzed the temperature variation of the transition energies by the Varshni equation ${ }^{11}$ and an empirical expression proposed by O'Donnel and Chen. ${ }^{12}$ The parameters that describe the temperature dependence of the band gap are evaluated and discussed. The broadening parameter is found to be insensitive to the temperature variation.

The carbon nitride films were deposited on clean Si (100) substrates in a microwave plasma assisted chemical vapor deposition (CVD) reactor. A mixture of semiconductor grade $\mathrm{NH}_{3}, \mathrm{CH}_{4}$, and $\mathrm{H}_{2}$ gases in various proportions was used as source gas, typical flow rates being 80-120 cubic centimeter per minute at STP (SCCM) for $\mathrm{H}_{2}, 80-1200$ SCCM for $\mathrm{NH}_{3}$, and 10-25 SCCM for $\mathrm{CH}_{4}$, while the chamber pressure was maintained at 90 Torr. The microwave power was $3.0-3.5 \mathrm{~kW}$ and the temperature of the substrates was held at $1000-1200{ }^{\circ} \mathrm{C}$, as measured by an optical pyrometer, during depositions. Heating of the substrates was achieved by the plasma itself. A significant incorporation of Si into the film was observed. $^{7,8}$ The presence of Si along with high substrate temperature promotes the formation of large crystallites. X-ray photoelectron spectroscopy (XPS) analyses of C (1s) and $\mathrm{N}(1 s)$ core levels suggest a multiple bonding structure between carbon and nitrogen atoms. The XPS analysis of the films, which enabled determination of the gross chemical composition, revealed the presence of a significant fraction of $\mathrm{Si}$ (about 15-20 at. \%) incorporated in the carbon nitride network. ${ }^{7,8}$ Microscopic investigation of the films reveals the coexistence of large grain $(>10 \mu \mathrm{m})$ and fine grain $(<1 \mu \mathrm{m})$ crystals. The crystals appear transparent when viewed 


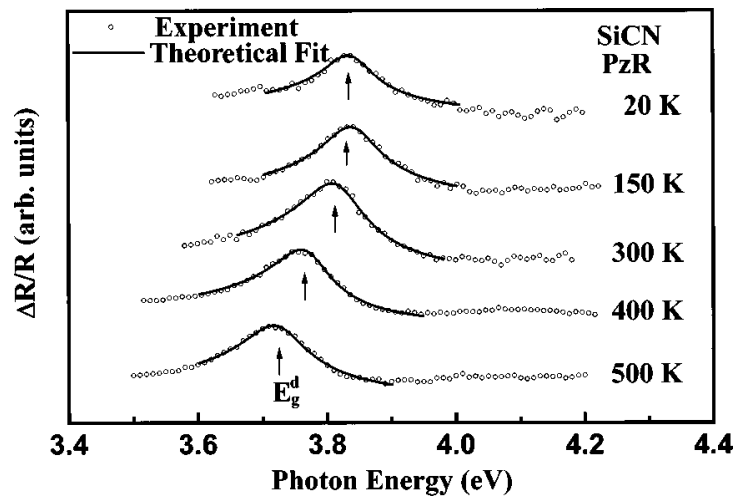

FIG. 1. The experimental PzR spectra (dotted curves) of SiCN at $20,150,300,400$, and $500 \mathrm{~K}$, respectively. The full curves are least-squares fits to the first derivative of a Lorentzian profile, which yields the direct band-to-band transition energy indicated by arrows.

through the optical microscope. The chemical composition of the crystals was determined from the scanning Auger depth profile data. The Si content varies from crystal to crystal and the Si atoms are believed to substitute for some of the C sites only. ${ }^{7,8}$ Preliminary structural studies suggest the presence of a crystalline carbon nitride compound corresponding to a hypothetical $\alpha-\mathrm{C}_{3} \mathrm{~N}_{4}$ phase, which may also be a stable hard material. ${ }^{13}$ The PzR method has been reported in the literature. ${ }^{9,10}$ The spot size of the monochromatic light was about $1 \mathrm{~mm}$ in diameter. The procedure for going to elevated temperatures is discussed in Ref. 14.

Displayed by the dotted curves in Fig. 1 are the PzR spectra in the vicinity of $E_{g}^{d}$ of a SiCN film for several temperatures between 20 and $500 \mathrm{~K}$. We have fitted the experimental line shape to the function ${ }^{15}$

$$
\frac{\Delta R}{R}=\operatorname{Re}\left[A e^{i \phi}\left(\Delta \varepsilon_{1}+i \Delta \varepsilon_{2}\right)\right],
$$

where $A$ and $\phi$ are the amplitude and phase of the feature, and $\Delta \varepsilon_{1}$ and $\Delta \varepsilon_{2}$ are the induced change of the real and imaginary parts of the dielectric function $\varepsilon$ due to external stress modulation. For interband transitions, the first derivative of the dielectric functions near the $M_{0}$ type of the threedimensional critical point is given by ${ }^{15}$

$$
\frac{d \varepsilon_{1}}{d E}=-\frac{d \varepsilon_{1}}{d E_{g}^{d}} \propto \frac{1}{2} \Gamma_{0}^{-1 / 2} F(-x), \quad \frac{d \varepsilon_{1}}{d \Gamma_{0}} \propto-\frac{1}{2} \Gamma_{0}^{-1 / 2} F(x)
$$

and

$$
\frac{d \varepsilon_{2}}{d E}=-\frac{d \varepsilon_{2}}{d E_{g}^{d}} \propto \frac{1}{2} \Gamma_{0}^{-1 / 2} F(x), \quad \frac{d \varepsilon_{2}}{d \Gamma_{0}} \propto \frac{1}{2} \Gamma_{0}^{-1 / 2} F(-x),
$$

where $F(x)=\left(x^{2}+1\right)^{-1 / 2}\left[\left(x^{2}+1\right)^{1 / 2}+x\right]^{1 / 2}$, with $x=(E$ $\left.-E_{g}^{d}\right) / \Gamma_{0}$, and $\Gamma_{0}$ is the broadening parameter of $E_{g}^{d}$. The solid curves in Fig. 1 show the least-squares fits to Eq. (1). The fits yield the parameters $E_{g}^{d}$ and $\Gamma_{0}$. The obtained values of $E_{g}^{d}$ are indicated by arrows in the figure.

From an analysis of the PzR spectrum at $300 \mathrm{~K}$, the direct band gap $E_{g}^{d}$ of $\mathrm{SiCN}$ is determined to be $3.81 \pm 0.01 \mathrm{eV}$. Liu and Cohen $^{3}$ have reported theoretical electronic bandstructure calculations of hypothetical $\beta-\mathrm{C}_{3} \mathrm{~N}_{4}$. Their calculations are based on the pseudopotential total-energy approach. ${ }^{16}$ The local density approximation (LDA), with the Wigner interpolation formula for the exchange and correlation functional, is used. The results indicate that the top of the valence band occurs along the $\Gamma$ to $A$ line and its separated from the bottom of the conduction band at $\Gamma$ by an indirect band gap of $3.2 \mathrm{eV}$. The states which lie between the valence-band maximum and about $5.3 \mathrm{eV}$ below the maximum correspond to nonbonding $\mathrm{N} p$ orbitals. The direct band gap at $\Gamma$ is about $4.0 \mathrm{eV}$. Despite the fact that our films may contain some unknown structures with a wide range of Si composition, ${ }^{7,8}$ our experimental value of the direct band gap nevertheless agreed reasonably well with the theoretical value for $\beta-\mathrm{C}_{3} \mathrm{~N}_{4}$. This good agreement may indicate that both $\alpha$ - and $\beta-\mathrm{C}_{3} \mathrm{~N}_{4}$ have direct band gaps close to each other. However, this point needs to be clarified by a similar theoretical calculation for $\alpha-\mathrm{C}_{3} \mathrm{~N}_{4}$ and will not be discussed further in this work.

Plotted in Fig. 2 are the temperature variations of the direct gap $E_{g}^{d}$ of $\mathrm{SiCN}$ film with representative error bars. A least-squares fit (the full curve) to the Varshni semiempirical relationship ${ }^{11}$

$$
E_{g}^{d}(T)=E(0)-\frac{\alpha T^{2}}{(\beta+T)}
$$

yields parameters as given in Table I. Here, $E(0)$ is the

TABLE I. Values of the Varshni- and O'Donnel-Chen-type fitting parameters, which describe the temperature dependence of the direct gaps of $\mathrm{SiCN}$ and $\mathrm{GaAs}$, and the indirect gaps of Si and diamond.

\begin{tabular}{lcccccc}
\hline \hline Material & $\begin{array}{c}E(0) \\
(\mathrm{eV})\end{array}$ & $\begin{array}{c}\alpha \\
\left(10^{-4} \mathrm{eV} / \mathrm{K}\right)\end{array}$ & $\begin{array}{c}\beta \\
(\mathrm{K})\end{array}$ & $\begin{array}{c}E_{g}(0) \\
(\mathrm{eV})\end{array}$ & $\begin{array}{c}\langle\hbar \omega\rangle \\
(\mathrm{meV})\end{array}$ \\
\hline $\mathrm{SiCN}$ & $3.831 \pm 0.01^{\mathrm{a}}$ & $-2.1 \pm 0.4^{\mathrm{a}}$ & $-1000 \pm 100^{\mathrm{a}}$ & $3.828 \pm 0.01^{\mathrm{a}}$ & $3.5 \pm 0.5^{\mathrm{a}}$ & $83 \pm 6^{\mathrm{a}}$ \\
$\mathrm{GaAs}$ & $1.5216^{\mathrm{b}}$ & $8.871^{\mathrm{b}}$ & $572^{\mathrm{b}}$ & $1.521^{\mathrm{c}}$ & $3.00^{\mathrm{c}}$ & $26.7^{\mathrm{c}}$ \\
$\mathrm{Si}$ & $1.1557^{\mathrm{b}}$ & $7.021^{\mathrm{b}}$ & $1108^{\mathrm{b}}$ & $1.170^{\mathrm{c}}$ & $1.49^{\mathrm{c}}$ & $25.5^{\mathrm{c}}$ \\
Diamond & $5.4125^{\mathrm{b}}$ & $-1.979^{\mathrm{b}}$ & $-1437^{\mathrm{b}}$ & $5.450^{\mathrm{c}}$ & $2.31^{\mathrm{c}}$ & $94.0^{\mathrm{c}}$ \\
\hline \hline
\end{tabular}

Present work.

${ }^{\mathrm{b}}$ Reference 11 .

${ }^{\mathrm{c}}$ Reference 12 . 


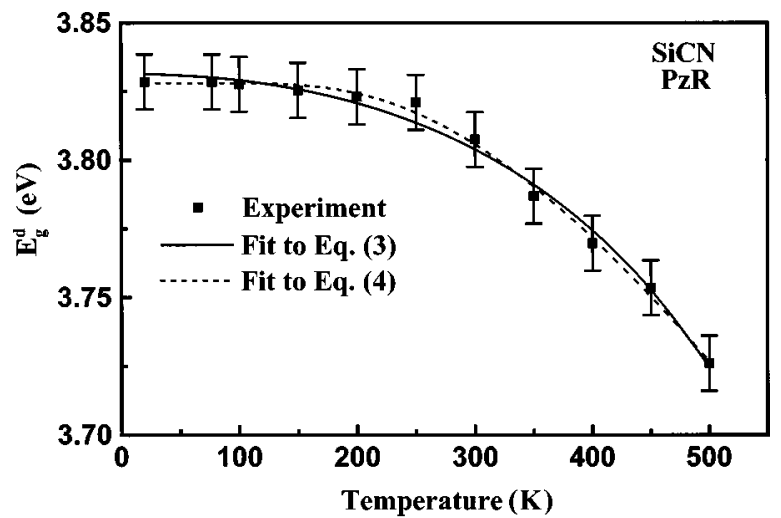

FIG. 2. The temperature dependence of the direct band gap $E_{g}^{d}$ of SiCN. Error bars are shown. The full curve is the least-squares fit to Eq. (3) and the dashed curve is the least-squares fit to Eq. (4).

direct band gap at absolute zero; $\alpha$ and $\beta$ are constants. The constant $\alpha$ is related to the electron-phonon interaction and $\beta$ is closely related to the Debye temperature. This fit is poor at low temperatures because Eq. (3) predicts a quadratic temperature dependence for the energy gap, whereas our experimental results indicate that the energy gaps are almost temperature independent at low temperatures. For comparison purposes we also have listed numbers for $\mathrm{GaAs}, \mathrm{Si}$, and diamond. ${ }^{11}$ It is difficult to explain the result that the fitted value of $\beta=-1000 \mathrm{~K}$. In general, one would expect the Debye temperature for semiconductors to be a positive quantity.

The data describing the temperature dependence of the direct energy gap $E_{g}^{d}$ of $\mathrm{SiCN}$ have also been fitted to an empirical expression proposed recently by O'Donnel and Chen, ${ }^{12}$

$$
E_{g}^{d}(T)=E_{g}(0)-S\langle\hbar \omega\rangle[\operatorname{coth}(\langle\hbar \omega\rangle / 2 k T)-1],
$$

where $E_{g}(0)$ is the energy gap at $0 \mathrm{~K}, S$ is a dimensionless coupling constant related to the strength of electron-phonon interaction, and $\langle\hbar \omega\rangle$ is an average phonon energy. The reasonably good fit obtained is shown in Fig. 2. It is statistically better than the Varshni fit. The values of the various parameters obtained are listed in Table I. For comparison purposes the numbers for previous report ${ }^{12}$ on $\mathrm{GaAs}, \mathrm{Si}$, and diamond are also included in Table I. As shown in Table I, our value of the electron-phonon coupling parameter $S$ is comparable to that of GaAs but much larger than those of $\mathrm{Si}$ and diamond.

From Eq. (4) we can write

$$
\frac{d E_{g}^{d}(T)}{d T}=-\frac{S\langle\hbar \omega\rangle^{2}}{2 k T^{2} \sinh ^{2}(\langle\hbar \omega\rangle / 2 k T)} .
$$

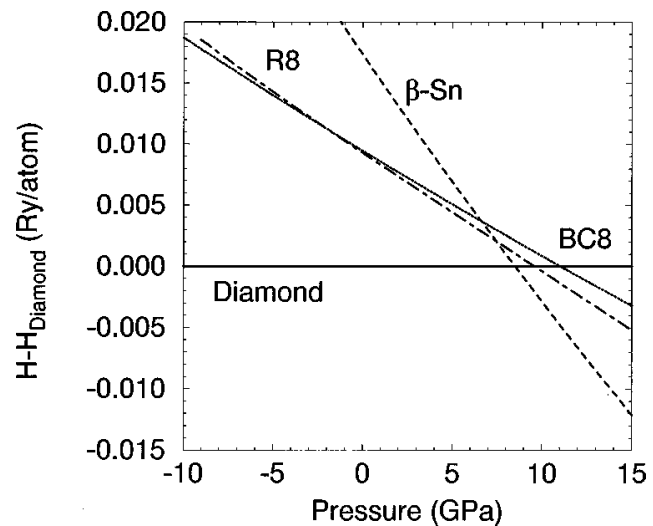

FIG. 3. The experimental temperature-dependent linewidth $\Gamma_{0}(T)$ of the band-to-band transitions. Error bars are shown.

At high temperatures, $k T$ is much larger than $\langle\hbar \omega\rangle$ and the slope of $E_{g}^{d}(T)$ vs $T$ curve approaches the limiting value $\left[d E_{g}^{d}(T) / T\right]_{\max }=-2 k S$. The calculated value of $\left[d E_{g}^{d}(T) / T\right]_{\max }$ is equal to $-0.60 \mathrm{meV} / \mathrm{K}$. The value of $\left[d E_{g}^{d}(T) / d T\right]$ as obtained from the linear extrapolation of our high-temperature $(400-500 \mathrm{~K})$ data is $-0.40 \mathrm{meV} / \mathrm{K}$. The discrepancy is due to the high average phonon energy $\langle\hbar \omega\rangle=83 \pm 6 \mathrm{meV}$ which is comparable with that of diamond and much larger than that of $\mathrm{GaAs}, \mathrm{Si}$, and most of the semiconductors. ${ }^{12}$ The large value of $\langle\hbar \omega\rangle$ may be a general characteristic of the $\mathrm{C}-\mathrm{N}$ films. However, a more systematic experimentation should be carried out to verify this property.

The experimental values of $\Gamma_{0}(T)$ for $E_{g}^{d}(T)$ as obtained from the line-shape fit for SiCN films are displayed in Fig. 3. The fitted values of $\Gamma_{0}$ are $\sim 45 \mathrm{meV}$ and nearly temperature independent. Both the large value of $\Gamma_{0}$ and its temperatureindependent characteristics might be related to the inhomogeneity of the polycrystalline films we have measured.

In conclusion, we have measured the temperature dependence of the direct band gap $E_{g}^{d}$ of SiCN films using PzR in the temperature range between 20 and $500 \mathrm{~K}$. At $300 \mathrm{~K} E_{g}^{d}$ is determined to be around $3.81 \mathrm{eV}$ in a good agreement with the theoretical calculation for $\beta-C_{3} \mathrm{~N}_{4}$. The temperature variations of $E_{g}^{d}$ have been analyzed by the Varshni and O'Donnel and Chen expressions. The average phonon energy obtained, $\langle\hbar \omega\rangle=83 \mathrm{meV}$, is comparable with that of diamond and much larger than that of most of the semiconductors. The temperature independence and large value of the broadening parameter might be related to the inhomogeneity of the polycrystalline samples.

The authors would like to acknowledge the support of the National Science Council of the Republic of China.
*Author to whom correspondence should be addressed. Electronic address: ysh@et.ntit.edu.tw

${ }^{1}$ H. Xin, C. Lin, S. Zhu, S. Zou, X. Shi, H. Zhu, and P. L. F. Hemment, Nucl. Instrum. Methods Phys. Res. B 103, 309 (1995).

${ }^{2}$ A. Y. Liu and M. L. Cohen, Science 245, 841 (1989).

${ }^{3}$ A. Y. Liu and M. L. Cohen, Phys. Rev. B 41, 10727 (1990).

${ }^{4}$ A. Y. Liu and R. M. Wentzcovithch, Phys. Rev. B 50, 10362 (1994).
${ }^{5}$ J. Ortega and O. F. Sankey, Phys. Rev. B 51, 2624 (1995).

${ }^{6}$ Y. Guo and W. A. Goddard III, Chem. Phys. Lett. 237, 72 (1995).

${ }^{7}$ L. C. Chen, C. Y. Yang, D. M. Bhusari, K. H. Chen, M. C. Liu, J. C. Lin, and T. J. Chuang, Diam. Relat. Mater. 5, 514 (1996).

${ }^{8}$ D. M. Bhusari, C. K. Chen, K. H. Chen, T. J. Chuang, L. C. Chen, and M. C. Lin, J. Mater. Res. 12, 322 (1997).

${ }^{9}$ F. H. Pollak and H. Shen, Mater. Sci. Eng. R. Rep. R10, 275 (1993).

${ }^{10}$ H. Mathieu, J. Allegre, and B. Gil, Phys. Rev. B 43, 2218 (1991). 
${ }^{11}$ Y. P. Varshni, Physica (Amsterdam) 34, 149 (1967).

${ }^{12}$ K. P. O'Donnel and X. Chen, Appl. Phys. Lett. 58, 2924 (1991).

${ }^{13}$ L. C. Chen, D. M. Bhusari, C. Y. Yang, K. H. Chen, T. J. Chuang, M. C. Lin, C. K. Chen, and Y. F. Huang (private communication).

${ }^{14}$ Y. S. Huang, H. Qiang, F. H. Pollak, G. D. Pettit, P. D. Kirchner,
J. M. Woodall, H. Stragier, and L. B. Sorensen, J. Appl. Phys. 70, 7537 (1991).

${ }^{15} \mathrm{P}$. Y. Yu and M. Cardona, Fundamentals of Semiconductors (Springer, Berlin, 1996), p. 307.

${ }^{16}$ M. L. Cohen, Phys. Scr. T1, 5 (1982). 